\title{
Design and Calibration of A Wearable Personal Distributed Exposimeter for LTE 800-2600 MHz Downlink Bands
}

\author{
Reza Aminzadeh, Arno Thielens, Patrick Van Torre, Sam Agneessens, \\ Matthias Van den Bossche, Hendrik Rogier, Luc Martens and Wout Joseph
}

\begin{abstract}
For the first time, a wearable personal distributed exposimeter (WPDE) is designed and calibrated for the LongTerm Evolution (LTE) 800 and 2600 MHz downlink bands. The proposed WPDE has a $68 \%$ confidence interval of 4.8-5.6 dB for different number of antennas and polarizations. Measurements of the WPDE are compared and validated with a commercial exposimeter in a real environment.
\end{abstract}

\section{INTRODUCTION}

Personal exposimeters (PEMs) are used to measure human exposure to radio-frequency (RF) electromagnetic fields. These portable devices are expected to measure the actual incident fields for which reference levels issued for instance by the International Commission on Non-Ionizing Radiation Protection (ICNIRP) [1]. The key problem with these devices is that they are calibrated in free space while used on body. This causes large measurement uncertainties and thus deviation of measured values form the actual incident fields [2].

Thielens et. al proposed a personal distributed exposimeter (PDE) for different frequency bands including GSM900DL [3] and WiFi-2 GHz [4] bands. To the best of authors' knowledge only commercial exposimeters are available in the market to measure exposure to Long-Term Evolution (LTE) signals and to date a personal distributed exposimeter-being capable of measuring personal exposure to LTE signals-has not been proposed.

In this paper, for the first time, we present design and calibration of a wearable personal distributed exposimeter (WPDE) for LTE 800 and $2600 \mathrm{MHz}$ downlink bands. The WPDE consists of 4 nodes (antennas) on the front and back of the user's body and measures the incident power densities for LTE downlink bands. The WPDE is calibrated on body in an anechoic chamber.

The methodology is described in Section II. Section III presents the results and Section IV concludes the paper.

\section{MATERIALS AND METHODS}

The WPDE consists of 1 master node that controls 4 nodes (antennas) covering both LTE 800 and $2600 \mathrm{MHz}$ downlink bands.

This research is funded under the French National Research Program for Environmental and Occupational Health of ANSES (2015/2 RF/07) as part of project ACCEDERA and by the Research Foundation Flanders (FWO-V) under grant agreement No FWO G003415N.

R. Aminzadeh, A. Thielens, P. Van Torre, S. Agneessens, M. Van den Bossche, H. Rogier, L. Martens and W. Joseph are with the Department of Information Technology (INTEC), Ghent University/imec Technologiepark-Zwijnaarde 15, B-9052 Ghent, Belgium. Email: reza.aminzadeh@ugent. be

\section{A. Nodes}

Each node consists of an elliptically polarized substrateintegrated-waveguide (SIW) textile antenna accompanied by a printed circuit board (PCB). All the antennas are fabricated using FR4 substrate and have a power reflection coefficient lower than $-10 \mathrm{~dB}$ in the desired frequency bands. An LTE $800 \mathrm{MHz}$ node is shown in Fig.1. Each node has a dynamic range of $80 \mathrm{~dB}$.

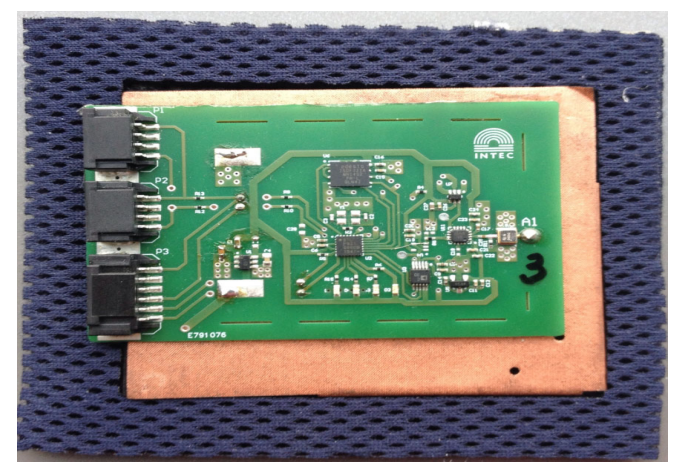

Fig. 1. An LTE $800 \mathrm{MHz}$ node (back side) including the textile antenna attached to the PCB.

The nodes are integrated into a jacket and are connected to the master node via a custom system bus protocol. The master node controls the whole system and functions as an interface between the user and the WPDE and similarly between the nodes and a PC to read/erase data.

\section{B. Combination of nodes}

In order to use multiple antennas per frequency band while keeping the number of measurements limited, 8 random locations are chosen on the front and back of the body. The selection criteria considers antenna placements on diametrically opposite locations on the body [5]. Figure 2 depicts the proposed locations for node placement on body. In the next step (Section II-C) calibration measurements are performed for each pair of nodes per frequency band and the location of nodes are optimized based on the results of on-body calibration measurements.

\section{On-body calibration}

Figure 3 demonstrates the on-body calibration setup in an anechoic chamber. The calibration measurements consist of two types of measurements: free space and on-body.

Firstly, using a Narda NBM-550 broadband field meter the free-space incident power densities $\left(S_{i n c}^{\text {free }, V}\right.$ and $S_{\text {inc }}^{\text {free,H}}$ ) 

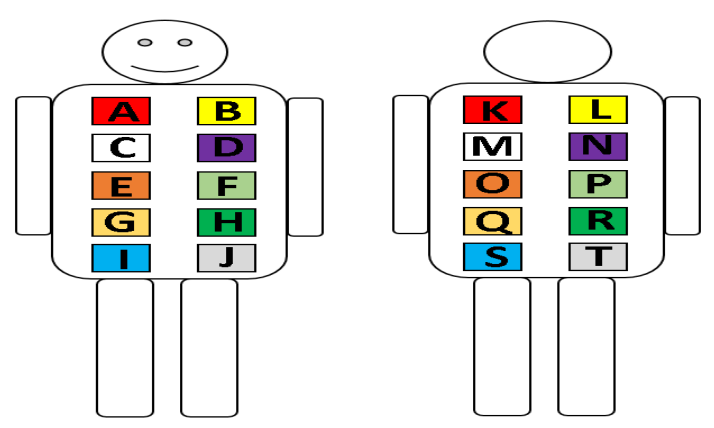

Fig. 2. The proposed nodes' locations on the front and back of the body.

are measured at different heights from the rotational platform $(54-202 \mathrm{~cm})$ for vertical and horizontal polarizations of the transmitting antenna (TX). During all the measurements the TX delivers a constant power of $30 \mathrm{~mW}$.

Secondly, the WPDE is worn by a 28 -year old male subject with height of $183 \mathrm{~cm}$ and mass of $79 \mathrm{~kg}$ and is positioned on a rotational platform (in the far field of the TX) in the anechoic room. Next, the subject is rotated over $360^{\circ}$ in the azimuthal angle for both polarizations of the TX. This results in a distribution for the registered electric field by each node $\left(P_{i}^{b o d y, H / V}\right)$ as a function of $\varphi(i=$ front/back $)$.

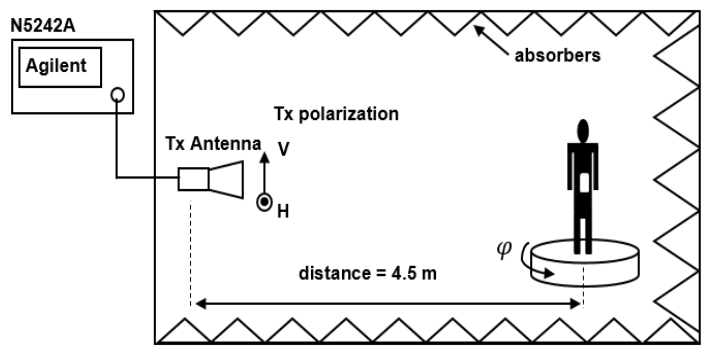

Fig. 3. On-body calibration measurement setup in the anechoic chamber.

In the last step, calibration results are used to calculate the on-body antenna aperture $A A_{i}$. Using a bootstrap method (100 iterations), $1000 \psi$ samples are drawn from a uniform distribution and the $A A_{i}$ values are determined for any realistic polarization as the sum of two orthogonal polarizations of the TX:

$$
\begin{aligned}
A A_{i}(\varphi, \psi)= & \frac{P_{i}^{b o d y, H}(\varphi)}{S_{i n c}^{f r e e, H}} \cos ^{2}(\psi) \\
& +\frac{P_{i}^{\text {body, },}(\varphi)}{S_{\text {inc }}^{\text {free }, V}} \sin ^{2}(\psi)
\end{aligned}
$$

During the measurements in a real environment a power $P_{i}^{\text {meas }}$ is recorded on each node $i$. This received power is used to determine the incident power density $\left(S_{i n c}\right)$ :

$$
S_{\text {inc }}=\frac{P_{i}^{\text {meas }}}{A A_{i}}
$$

where $A A_{i}$ is the effective median on-body aperture obtained from (1).

\section{Measurement in real environment}

The subject equipped with the WPDE and a commercial exposimeter (EME SPY 200 on the right hip) walks along a predefined path (Fig. 4) in Campus Ardoyen, Ghent University, Belgium. The route (thick gray line) is approximately 1 $\mathrm{km}$ long and lasts 15 minutes.

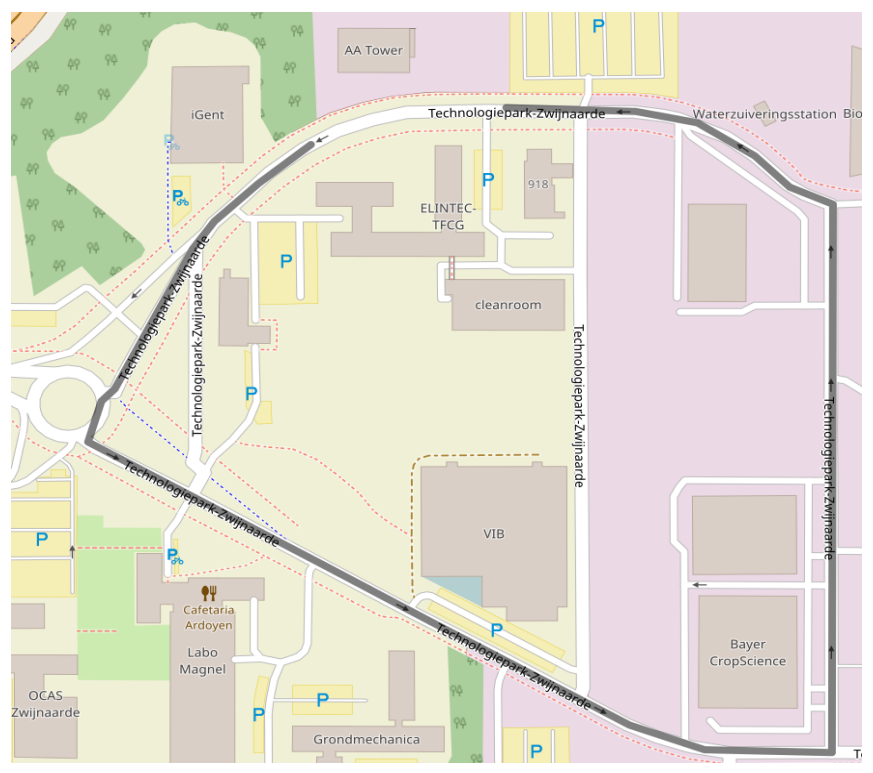

Fig. 4. Path followed by the subject wearing the WPDE and EME SPY200 in Campus Ardoyen, Ghent University, Belgium.

\section{RESULTS AND DISCUSSION}

Figure 5 shows the minimal $68 \%$ confidence interval (CI68) of the on-body antenna aperture obtained from calibration measurements. Single nodes (front or back) have the highest $C I 68$ values. LTE $800 \mathrm{MHz}$ has the lowest $C I 68$ values: $12.38 \mathrm{~dB}$ for $Q_{V}$ and $12.84 \mathrm{~dB}$ for $P_{H}$. Using the average over front and back improved the results. For LTE $800 \mathrm{MHz}$, nodes CQ show an improvement of 7.6$11 \mathrm{~dB}$ while HP has an improvement of 7-9.6 dB. For LTE $2600 \mathrm{MHz}$ the improvement for using two antennas is in the range of 19-20.4 dB for nodes BT and 19-21.1 dB for nodes CM. Also the combination of $C_{H}, Q_{V}$ and $B_{H}, T_{V}$ have the minimum CI68 for LTE $800 \mathrm{MHz}$ and $2600 \mathrm{MHz}$, respectively. Therefore, these two combinations are used as the optimized combination of four nodes for the WPDE. The median on-body antenna aperture for these optimized combinations are $9.81 \mathrm{~cm}^{2}$ (LTE $800 \mathrm{MHz}$ ) and $2.92 \mathrm{~cm}^{2}$ (LTE $2600 \mathrm{MHz}$ ).

Figure 6 shows the measured incident power density for the LTE $800 \mathrm{MHz}$ (dominant signal) during the pre-defined walk using the WPDE and the EME Spy 200. The incident power density on the WPDE is determined after applying the on-body antenna aperture to the received powers on the optimized nodes during the walk. The measured median 


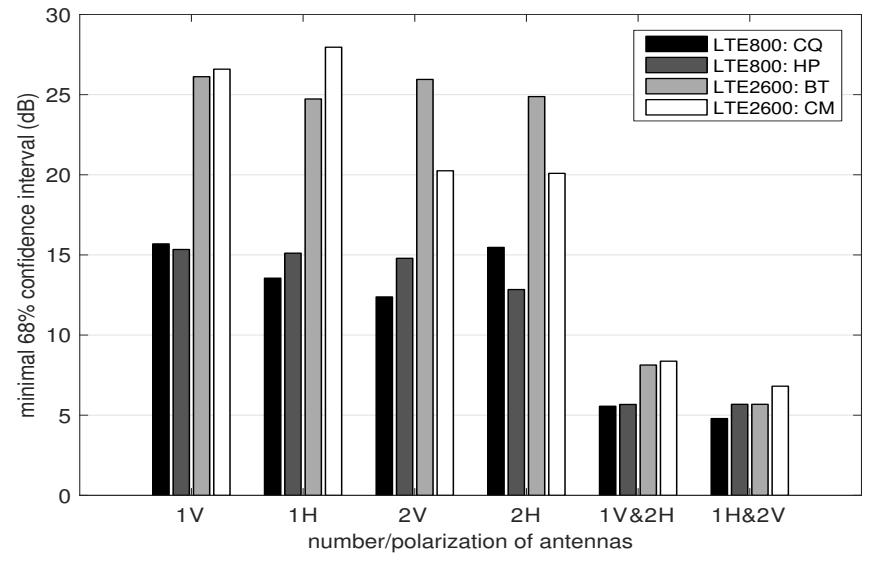

Fig. 5. Minimal $68 \%$ confidence interval of the on-body antenna aperture for both LTE downlink bands for different number of antennas and polarizations.

$S_{\text {inc }}$ is $-60.6 \mathrm{dBW} / \mathrm{m}^{2}$ for the EME SPY 200 and $53.92 \mathrm{dBW} / \mathrm{m}^{2}$ for the WPDE. This is a difference of $6.7 \mathrm{~dB}$ and shows that EME SPY 200 underestimates the actual power densities as its performance is affected by shadowing of the body. Also the difference in the measured values by both devices is due to the fact that EME SPY 200 is not calibrated on the subject. The maximum $S_{\text {inc }}$ measured by the WPDE during the walk is $51 \mathrm{~mW} / \mathrm{m}^{2}$.

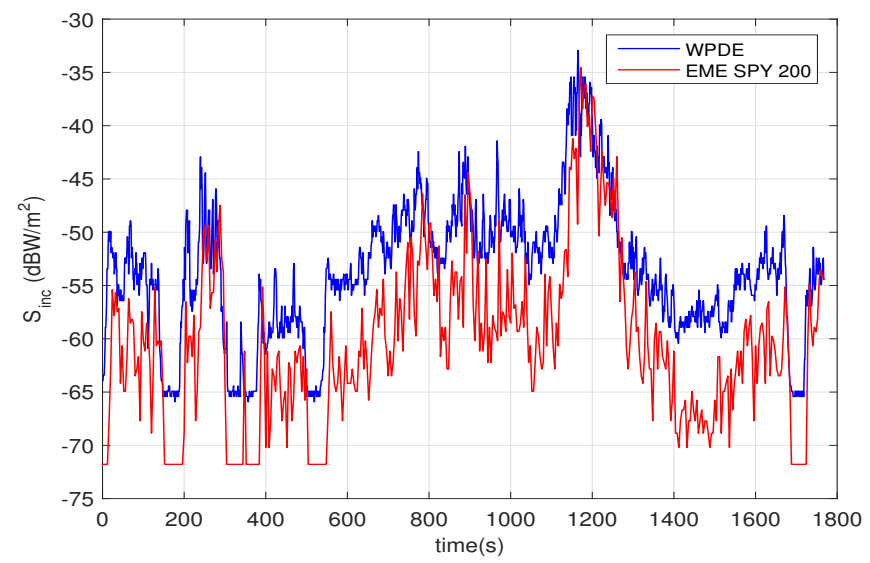

Fig. 6. Measured incident power density on the body, measured with the optimized nodes of the WPDE vs EME SPY 200 for LTE $800 \mathrm{MHz}$.

\section{CONCLUSION}

For the first time, a wearable personal distributed exposimeter is designed and calibrated for LTE 800 and $2600 \mathrm{MHz}$ downlink bands. The WPDE is calibrated on a male subject in an anechoic chamber and the location of nodes are optimized on the body. Using two nodes for each frequency band an improvement of 7.6 to $21.1 \mathrm{~dB}$ is obtained for the $68 \%$ confidence interval of the on body antenna aperture. The WPDE has an on-body antenna aperture of $9.81 \mathrm{~cm}^{2}$ and $2.92 \mathrm{~cm}^{2}$ for LTE 800 and $2600 \mathrm{MHz}$, respectively. The subject wearing the WPDE and an EME
SPY 200, followed a pre-defined walk in Ghent, Belgium. The results shows that EME SPY 200 underestimate the personal exposure to LTE signals. During the measurement campaign a median $S_{i n c}-53.92 \mathrm{dBW} / \mathrm{m}^{2}$ is registered for the WPDE. The maximum $S_{\text {inc }}$ measured by the WPDE during the walk is $51 \mathrm{~mW} / \mathrm{m}^{2}$.

\section{REFERENCES}

[1] International Commission on Non-ionizing Radiation Protection (ICNIRP), "Guidelines for limiting exposure to time-varying electric, magnetic, and electromagnetic fields (up to $300 \mathrm{GHz}$ )," Health Physics, vol. 74, no. 4, pp. 494-522, 1998.

[2] J. F. Bolte, G. van der Zande, and J. Kamer, "Calibration and uncertainties in personal exposure measurements of radiofrequency electromagnetic fields," Bioelectromagnetics, vol. 32, no. 8, pp. 652663, 2011.

[3] P. Vanveerdeghem, P. V. Torre, A. Thielens, J. Knockaert, W. Joseph, and H. Rogier, "Compact personal distributed wearable exposimeter," IEEE Sensors Journal, vol. 15, no. 8, pp. 4393-4401, 2015.

[4] A. Thielens, S. Agneessens, H. De Clercq, J. Lecoutere, L. Verloock, E. Tanghe, S. Aerts, R. Puers, H. Rogier, L. Martens, et al., "On-body calibration and measurements using a personal, distributed exposimeter for wireless fidelity," Health physics, vol. 108, no. 4, pp. 407-418, 2015.

[5] A. Thielens, P. Vanveerdeghem, P. Van Torre, S. Gngler, M. Rsli, H. Rogier, L. Martens, and W. Joseph, "A personal, distributed exposimeter: Procedure for design, calibration, validation, and application," Sensors, vol. 16, no. 2,2016 